\title{
L'invention de la vie privée et le modèle d'Horace, sous la direction de B. Delignon, N. Dauvois et L. Cottegnies
}

\section{Maurizio Busca}

\section{(2) OpenEdition}

\section{Journals}

\section{Edizione digitale}

URL: https://journals.openedition.org/studifrancesi/20996

DOI: 10.4000/studifrancesi.20996

ISSN: 2421-5856

\section{Editore}

Rosenberg \& Sellier

\section{Edizione cartacea}

Data di pubblicazione: 1 décembre 2019

Paginazione: 558

ISSN: 0039-2944

\section{Notizia bibliografica digitale}

Maurizio Busca, «L'invention de la vie privée et le modèle d'Horace, sous la direction de B. Delignon, N Dauvois et L. Cottegnies», Studi Francesi [Online], 189 (LXIII | III) | 2019, online dal 01 mars 2020, consultato il 11 novembre 2021. URL: http://journals.openedition.org/studifrancesi/20996 ; DOl: https://doi.org/10.4000/studifrancesi.20996

Questo documento è stato generato automaticamente il 11 novembre 2021.

\section{cc) (†)}

Studi Francesi è distribuita con Licenza Creative Commons Attribuzione - Non commerciale - Non opere derivate 4.0 Internazionale. 


\title{
L'invention de la vie privée et le modèle d'Horace, sous la direction de B. Delignon, N. Dauvois et L. Cottegnies
}

\author{
Maurizio Busca
}

\section{NOTIZIA}

L'invention de la vie privée et le modèle d'Horace, sous la direction de B. Delignon, N.

Dauvois et L. Cottegnies, Paris, Classiques Garnier, 2017, «Rencontres» 261, 477 pp.

1 L'espressione della tensione fra sfera pubblica e privata, fra impegno civile e autonomia individuale e fra mecenate e artista trova nell'opera di Orazio un modello destinato a conoscere una straordinaria fortuna nel Rinascimento europeo. Alla posterità delle forme, dello stile e della persona che Orazio adotta nella rappresentazione del poeta priuatus, e più latamente all'emergere di una letteratura attenta all'esplorazione della dimensione dell'intimità è stato dedicato il convegno di Parigi (17-19 ottobre 2013) i cui atti sono pubblicati nel presente volume. Un primo nucleo di interventi è incentrato su aspetti puntuali dell'opera oraziana e della sua ricezione alta; seguono gli studi sulla sua ricezione nella prima modernità in Francia, Inghilterra, Italia e Spagna. Questi i contributi raccolti.

2 Line Cottegnies, Nathalie Dauvois et Bénédicte Delignon, Introduction, pp. 7-14; Marie Ledentu, Horace et le discours autobiographique. L'empreinte littéraire de l'histoire politique des années 40-30 av. J.-C., pp. 15-30; Alain Deremetz, Horatius Personatus, p. 31-42; Emily Gowers, Rus in urbe: l'intimité urbaine dans la poésie d'Horace, pp. 43-57; Mario Citroni, Images de la vie privée dans les "Épitres" d'Horace. Ambiguité d'un modèle éthique, pp. 59-76; Bénédicte Delignon, Dîner avec Mécène. Vie privée et vie publique dans les "Satires" et dans les "Odes", pp. 77-90; Clément Auger, Priuatus chez Horace et dans sa première réception, échos d'un mot, pp. 91-107; Perrine Galand, Jean Salmon Macrin peintre de sa vie privée. De l'imitation d'Horace et d'autres à l'imprévisible découverte de soi, pp. 109-122; Jean Lecointe, 
Modèle horatien et "privatisation» de la plainte funèbre entre 1530 et 1550. Autour de "La Navire" de Marguerite de Navarre, pp. 123-142; André Bayrou, La vie privée des serviteurs de l'État. Échos horatiens dans les épîtres de Michel de L'Hospital (ca. 1506-1573), pp. 143-163; Bruno Méniel, "Les Regrets" et le sermo horatien. Une poésie de l'intime, pp. 165-182; Nicolas Lombart, Formes et fonctions de l'autodérision chez Ronsard. Le modèle horatien, pp. 183-201; Marie-Claire Thomine-Bichard, Du Fail, Horace et la vie privée, pp. 203-225; Bénédicte Boudou, Le style mêlé dans les "Histoires tragiques" de la fin du $\mathrm{XVI}^{\mathrm{e}}$ siècle. Boaistuau, Poissenot, Habanc, Belleforest, pp. 227-242; Pascal Debailly, Lyrisme satirique et sublime de l'intime chez Horace et ses disciples, pp. 243-257; Michel Magnien, Horace, Montaigne et l'«arrière-boutique», pp. 259-275; Hélène Merlin-Kajman, Le privé et l'intimité au XVII siècle doivent-ils quelque chose à Horace?, pp. 277-298; Alessandra Villa, L'Arioste qui voulait être Horace, pp. 299-320; Clara Marías Martínez, Imitation of Horace and the Construction of a Private Personality. A Case-Study Diego Hurtado de Mendoza's Verse Epistles, pp. 321-337; Anne-Marie Miller-Blaise, Du for privé et de sa publicité. Portraits horatiens de l'artiste dans les "Satires" de John Donne, pp.339-357; Marie-Alice Belle, Autour de la question des «communautés de lecteurs». Discours liminaire et amicitia horatienne chez Jonson et ses contemporains, pp. 359-380; Line Cottegnies, Robert Herrick, un Horace anglais? La voix du poète dans "Hesperides" (1648), pp. 381-392; Christopher de Warrenne Waller, Horatian Motifs in Andrew Marvell's Poetry. Public Action and Private Retreat, pp. 393-410; Chantal Schütz, Le "Micro-cynicon" de Thomas Middleton (1598). Un exercice d'imitatio trop réussi?, pp. 411-424.

3 Numerosi saggi guardano al Cinquecento francese, sia neolatino che vernacolare. Lo studio di Galand sul recupero del modello oraziano nelle raccolte poetiche di Jean Salmon, che in Orazio trova un maestro dichiarato, permette di osservare la parte di originalità del poeta neolatino: nell'opera di Salmon l'opposizione fra i piaceri della vita privata e i doveri della vita di corte si nutre infatti di elementi autobiografici (legati in particolare all'esperienza famigliare e all'ispirazione religiosa) che motivano sperimentazioni linguistiche e stilistiche. Il confronto con Orazio conduce a esiti ben diversi nelle epistole di Michel de l'Hospital: benché il poeta latino costituisca un punto di riferimento sicuro per L'Hospital, Bayrou rileva come la ripresa formale del modello classico sia spesso funzionale al rifiuto dell'ideologia che vi si trova espressa e, di converso, alla celebrazione della partecipazione attiva alla vita politica. Il contributo di Lecointe è dedicato a Marguerite de Navarre di cui, oltre alla riscrittura di Odi, III, 9 (che fornisce il modello del Dialogue: Regulus et Lucia), l'A. analizza il poema La navire nel contesto della ridefinizione del genere del lamento funebre fra gli anni trenta e cinquanta del Cinquecento, riconoscendovi la presenza - mediata - di tratti oraziani. Le opere del poeta di Venosa appaiono in filigrana anche nei Regrets di Du Bellay e nel Second livre de Meslanges di Ronsard, come mostrano i lavori di Méniel e Lombart. Per quanto riguarda Du Bellay, al di là delle puntuali riprese testuali, Méniel osserva le affinità che lo stile, i temi e il trattamento della dimensione dell'intimità nei Regrets presentano con il sermo delle Satire. Per quanto riguarda Ronsard, Lombart prende in esame gli episodi di autoderisione presenti nella raccolta di Meslanges del 1559: anche in questo caso, non sono tanto tracce di rapporti intertestuali manifesti a emergere, quanto una trama di motivi e tratti discorsivi condivisi che partecipano alla costruzione dell'ethos dei due poeti. Citazioni, parafrasi e allusioni oraziane punteggiano invece le opere narrative di Noël Du Fail (Propos rustiques, Baliverneries d'Eutrapel et Contes et discours d'Eutrapel): Thomine propone una topologia delle riprese testuali e sottolinea come il poeta latino, di cui due citazioni sono poste in esergo ai Contes et discours, 
fornisca all'opera della maturità di Du Fail un tono (la vivacità), uno stile (il sermo cotidianus) e un tema (l'otium) di riferimento. Un secondo saggio sulla narrativa cinquecentesca guarda invece al corpus delle histoires tragiques: in queste pagine, Boudou si interroga sui legami fra sermo cotidianus oraziano, style mêlé e scrittura della vita privata nelle opere di quattro dei maggiori esponenti del genere. Magnien, infine, si interessa all'influenza del modello oraziano sull'opera e sulla vita di Montaigne, in particolare per quel che concerne la rinuncia agli incarichi pubblici e il ritiro a vita privata. 